\title{
On (strong) $\alpha$-favorability of the Wijsman hyperspace
}

\author{
Leszek Piątkiewicz, László Zsilinszky* \\ Department of Mathematics and Computer Science, University of North Carolina at Pembroke, Pembroke, NC 28372, USA
}

\section{A R T I C L E I N F O}

\section{Article history:}

Received 5 February 2010

Received in revised form 19 July 2010

Accepted 31 July 2010

\section{Keywords:}

Wijsman topology

Ball topology

Baire space

Banach-Mazur game

Strong Choquet game

(Strongly) $\alpha$-favorable space

Bernstein set

Baire metric

\begin{abstract}
A B S T R A C T
The Banach-Mazur game as well as the strong Choquet game are investigated on the Wijsman hyperspace from the nonempty player's (i.e. $\alpha$ 's) perspective. For the strong Choquet game we show that if $X$ is a locally separable metrizable space, then $\alpha$ has a (stationary) winning strategy on $X$ iff it has a (stationary) winning strategy on the Wijsman hyperspace for each compatible metric on $X$. The analogous result for the Banach-Mazur game does not hold, not even if $X$ is separable, as we show that $\alpha$ may have a (stationary) winning strategy on the Wijsman hyperspace for each compatible metric on $X$, and not have one on $X$. We also show that there exists a separable 1st category metric space such that $\alpha$ has a (stationary) winning strategy on its Wijsman hyperspace. This answers a question of Cao and Junnila (2010) [6].
\end{abstract}

(c) 2010 Elsevier B.V. All rights reserved.

\section{Introduction}

There has been a considerable effort in exploring various completeness properties of the Wijsman hyperspace topology $\tau_{w(d)}$, i.e. the weak topology on the nonempty closed subsets $C L(X)$ of the metric space $(X, d)$ generated by the distance functionals viewed as functions of set argument [24]. The Wijsman topology is a fundamental tool in the construction of the lattice of hyperspace topologies, for many studied hypertopologies arise as suprema and infima, respectively, of appropriate Wijsman topologies (cf. $[4,11])$.

It was first shown by Effros [13], that a Polish space admits a metric for which the Wijsman topology is Polish; later, Beer showed [3,4], that given a separable complete metric base space, the corresponding Wijsman hyperspace is Polish. Finally, Costantini demonstrated in [9], that Polish base spaces always generate Polish Wijsman topologies (a short proof, using the so-called strong Choquet game, was found by the second author in [26]). As a related result, note that the Wijsman hyperspace is analytic iff $X$ is analytic [2].

Beer asked, whether complete metrizability of $X$ alone (without separability) is equivalent to some completeness property of the Wijsman hyperspace. Costantini [10] showed that a natural candidate, Čech-completeness, is not the right property; on the other side, complete metrizability of $X$ guarantees Baireness [25], even strong $\alpha$-favorability [26], of the Wijsman hyperspace regardless of the underlying metric on $X$. It is also known, that less than complete metrizability of $X$ - e.g. having a dense completely metrizable subspace [27] or being an almost locally separable Baire space [28], or being hereditarily Baire [7], respectively - guarantees Baireness of the Wijsman topology; however, $\tau_{w(d)}$ may be non-hereditarily Baire, even if $X$ is separable, hereditarily Baire and has a dense completely metrizable subspace [27], or $X$ is completely metrizable [8], respectively. All these results suggested that at least as strong a completeness property is needed on the base space as one wants to impose on the hypertopology, so it was somewhat surprising to find a separable 1 st category metric

\footnotetext{
* Corresponding author.

E-mail addresses: leszek@uncp.edu (L. Piątkiewicz), laszlo@uncp.edu (L. Zsilinszky).
} 
space with a Baire Wijsman hyperspace [28,29]. This result was recently strengthened in [6] by showing that the example of [28] and an other 1st category metric space give rise to a Wijsman topology with various Amsterdam properties.

It is the purpose of this paper to continue this research by investigating $\alpha$-favorability, and strong $\alpha$-favorability, respectively, of $\tau_{w(d)}$ which are completeness properties associated with the so-called Banach-Mazur game, and the strong Choquet game, respectively (see Section 3 for definitions and basic results about these games). We partially answer Beer's question for locally separable spaces in Theorem 4.1, and we show that this characterization may break down in spaces that are not locally separable (Theorem 4.2 ).

It has been shown in [28], that if $X$ is an almost locally separable metrizable space, and $\tau_{w(d)}$ is not $\beta$-favorable for each compatible metric $d$ on $X$, then $X$ is not $\beta$-favorable. In Theorem 5.5 we show that if we replace non $\beta$-favorability with the stronger property of $\alpha$-favorability, the corresponding statement is not true, not even for a separable $X$. We answer in the positive Question 4.2 of [6] about almost countable base-compactness of $\tau_{w(d)}$.

\section{Preliminaries}

Let $X$ be a metrizable space and $d$ a compatible metric on $X$. Denote by $C L(X)$ the set of all nonempty closed subsets of $X$, and by $C B(X)$ (resp. $O B(X)$ ), the proper closed (resp. open) balls $B(x, \varepsilon)=\{y \in X: d(x, y) \leqslant \varepsilon\}$ (resp. $S(x, \varepsilon)=\{y \in$ $X: d(x, y)<\varepsilon\})$, where $\varepsilon>0, x \in X$. For any $S \subseteq X$ denote

$$
S^{-}=\{A \in C L(X): A \cap S \neq \emptyset\} \text { and } S^{+}=\{A \in C L(X): A \subseteq S\} .
$$

The Wijsman topology $\tau_{w(d)}$ on $C L(X)$ is the weak topology generated by the distance functionals $d(x, A)=\inf _{a \in A} d(x, a)$ $(A \in C L(X), x \in X)$, viewed as functions of the set argument.

The ball topology $\tau_{b(d)}$ on $C L(X)$ has as a subbase sets of the form $V^{-}$, where $V$ is an open subset of $X$, plus sets of the form $\left(B^{c}\right)^{+}$, where $B$ is a finite union of members of $C B(X) \cup\{\varnothing\}$ (here, $B^{c}$ is the complement of $B$ ). It is not hard to see, that a base $\mathcal{B}_{d}$ for the ball topology consists of the sets

$$
\left\langle V_{0}, \ldots, V_{n}\right\rangle_{B}=\left(B^{c}\right)^{+} \cap \bigcap_{i \leqslant n} V_{i}^{-},
$$

where $B$ is a finite union of members of $C B(X) \cup\{\emptyset\}$, and $V_{0}, \ldots, V_{n} \subseteq X$ are pairwise disjoint open sets missing $B$ ( $\left.n<\omega\right)$. We will also use the notation $\langle\mathcal{V}\rangle_{B}$, where $\mathcal{V}=\left\{V_{0}, \ldots, V_{n}\right\}$. See [16] for a characterization of the coincidence of the ball and the Wijsman topologies.

With a slight modification of [20, Lemma 2.3.1], it is not hard to show

Theorem 2.1. The following are equivalent:

(i) $\left\langle U_{0}, \ldots, U_{m}\right\rangle_{D} \subseteq\left\langle V_{0}, \ldots, V_{n}\right\rangle_{B}$

(ii) $B \subseteq D$ and $\forall i \leqslant n \exists j \leqslant m$ with $U_{j} \subseteq V_{i}$.

\section{Topological games}

Let $X$ be a topological space, and $\mathcal{P}$ a fixed $\pi$-base. The Banach-Mazur game $B M(X)$ is played as follows: players $\beta$, and $\alpha$ alternate in choosing elements of $\mathcal{P}$, with $\beta$ choosing first, so that $B_{0} \supseteq A_{0} \supseteq B_{1} \supseteq A_{1} \supseteq \cdots \supseteq B_{n} \supseteq A_{n} \supseteq \cdots$. Then $B_{0}, A_{0}, \ldots, B_{n}, A_{n}, \ldots$ is a play in $B M(X)$, and $\alpha$ wins this play if $\bigcap_{n<\omega} A_{n}\left(=\bigcap_{n<\omega} B_{n}\right) \neq \emptyset$, otherwise, $\beta$ wins. A strategy in $B M(X)$ is a function $\sigma: \mathcal{P}^{<\omega} \rightarrow \mathcal{P}$ such that $\sigma\left(W_{0}, \ldots, W_{n}\right) \subseteq W_{n}$ for all $n<\omega$, and $\left(W_{0}, \ldots, W_{n}\right) \in \mathcal{P}^{n+1}$. A tactic in $B M(X)$ is a function $t: \mathcal{P} \rightarrow \mathcal{P}$ such that $t(W) \subseteq W$ for all $W \in \mathcal{P}$. A winning strategy (resp. winning tactic) for $\alpha$ is a strategy (tactic) $\sigma$ such that $\alpha$ wins every play of $B M(X)$ compatible with $\sigma$, i.e. such that $\sigma\left(B_{0}, \ldots, B_{n}\right)=A_{n}$ (resp. $\sigma\left(B_{n}\right)=A_{n}$ ) for all $n<\omega$. A winning strategy (resp. winning tactic) for $\beta$ is defined analogously. The space $X$ is called (weakly) $\alpha$-favorable [22], if $\alpha$ has a winning tactic (resp. winning strategy) in $B M(X)$ (these properties are not equivalent in general - see [12]). A space $X$ is called $\beta$-favorable, if $\beta$ has a winning strategy in $B M(X)$ (this is equivalent to $\beta$ having a winning tactic in $B M(X)$ - see [15]).

It is known that if $X$ is metrizable, then $X$ is (weakly) $\alpha$-favorable iff $X$ contains a dense completely metrizable subspace [23]; on the other side, a topological space $X$ is not $\beta$-favorable iff $X$ is a Baire space (i.e. each sequence of dense open subsets of $X$ intersects in a dense subset of $X$ - see [19]).

A mapping $f$ from a topological space $X$ onto a topological space $Y$ is said to be feebly continuous (feebly open) iff int $f^{-1}(V) \neq \varnothing$ (int $\left.f(U) \neq \emptyset\right)$ for any nonempty open $V \subset Y(U \subset X)$. A feeble homeomorphism is a feebly continuous feebly open bijection [17].

Proposition 3.1. Let $f: X \rightarrow Y$ be a feeble homeomorphism. Then $X$ is (weakly) $\alpha$-favorable if and only if $Y$ is (weakly) $\alpha$-favorable.

Proof. Let $\sigma_{X}$ be a winning strategy (tactic) for $\alpha$ in $B M(X)$. Define a strategy (tactic) $\sigma_{Y}$ for $\alpha$ in $B M(Y)$ as follows: given $Y$-open sets $V_{0}, \ldots, V_{k}$ (resp. $Y$-open $V$ ), let 


$$
\sigma_{Y}\left(V_{0}, \ldots, V_{k}\right)=\operatorname{int}_{Y}\left(f\left(\sigma_{X}\left(\operatorname{int}_{X} f^{-1}\left(V_{0}\right), \ldots, \operatorname{int}_{X} f^{-1}\left(V_{k}\right)\right)\right)\right)
$$

(resp. $\left.\sigma_{Y}(V)=\operatorname{int}_{Y}\left(f\left(\sigma_{X}\left(\operatorname{int}_{X} f^{-1}(V)\right)\right)\right)\right)$. If $V_{0}, \ldots, V_{k}, \ldots$ is a run of $B M(Y)$ compatible with $\sigma_{Y}$, then $\operatorname{int}_{X} f^{-1}\left(V_{0}\right), \ldots$, int $_{X} f^{-1}\left(V_{k}\right), \ldots$ is a run of $B M(X)$ compatible with $\sigma_{X}$, thus, we can find some $x \in \bigcap_{k} \operatorname{int}_{X} f^{-1}\left(V_{k}\right)$. Then $f(x) \in \bigcap_{k} V_{k}$, so $\sigma_{Y}$ is a winning strategy (tactic) for $\alpha$ in $B M(Y)$. The equivalence now follows since $f^{-1}: Y \rightarrow X$ is a feeble homeomorphism, too.

Let $\mathcal{B}$ be a base for $X$, and denote

$$
\mathcal{E}=\mathcal{E}(X)=\mathcal{E}(X, \mathcal{B})=\{(x, U) \in X \times \mathcal{B}: x \in U\} .
$$

The strong Choquet game $\operatorname{Ch}(X)$ is played similarly to the Banach-Mazur game, but in addition to the open $B, \beta$ also chooses a point $x \in B$. More precisely, players $\beta$ and $\alpha$ alternate in choosing $\left(x_{n}, B_{n}\right) \in \mathcal{E}$ and $A_{n} \in \mathcal{B}$, respectively, with $\beta$ choosing first so that for each $n<\omega, x_{n} \in A_{n} \subseteq B_{n}$, and $B_{n+1} \subseteq A_{n}$. The play $\left(x_{0}, B_{0}\right), A_{0}, \ldots,\left(x_{n}, B_{n}\right), A_{n}, \ldots$ is won by $\alpha$, if $\bigcap_{n<\omega} A_{n}\left(=\bigcap_{n<\omega} B_{n}\right) \neq \emptyset$; otherwise, $\beta$ wins. A strategy in $\operatorname{Ch}(X)$ for $\alpha$ is a function $\sigma: \mathcal{E}^{<\omega} \rightarrow \mathcal{B}$ such that $x_{n} \in \sigma\left(\left(x_{0}, B_{0}\right), \ldots,\left(x_{n}, B_{n}\right)\right) \subseteq B_{n}$ for all $\left(\left(x_{0}, B_{0}\right), \ldots,\left(x_{n}, B_{n}\right)\right) \in \mathcal{E}^{<\omega}$. A tactic in $\operatorname{Ch}(X)$ for $\alpha$ is a function $t: \mathcal{E} \rightarrow \mathcal{B}$ such that $x \in t(x, B) \subseteq B$ for all $(x, B) \in \mathcal{E}$. Winning strategies and tactics in $\operatorname{Ch}(X)$ are defined similarly to the ones for the Banach-Mazur game.

The space $X$ is strongly $\alpha$-favorable [22] (resp. strongly Choquet [19]), provided $\alpha$ has a winning tactic (resp. winning strategy) in $C h(X)$. Choquet's Theorem (see [5,19]) claims, that a metrizable space is strongly $\alpha$-favorable, and equivalently, strongly Choquet iff it is completely metrizable; however, $\alpha$ having a winning strategy or tactic in $\operatorname{Ch}(X)$ is not necessarily equivalent in nonmetrizable spaces (the example in [12] is strongly Choquet and not $\alpha$-favorable).

\section{Strong $\alpha$-favorability of the Wijsman topology}

The celebrated Beer-Costantini Theorem claims that $\left(C L(X), \tau_{w(d)}\right)$ is Polish iff $(X, d)$ is Polish $[3,9]$. Since in a metrizable space, complete metrizability is equivalent to strong $\alpha$-favorability, we can rephrase this theorem as follows:

Theorem B-C. Let $X$ be a separable metrizable space. Then the following are equivalent:

(i) $\left(C L(X), \tau_{w(d)}\right)$ is strongly $\alpha$-favorable for each compatible metric $d$ on $X$,

(ii) $X$ is completely metrizable.

It turns out that separability can be weakened to local separability:

Theorem 4.1. Let $X$ be a locally separable metrizable space. Then the following are equivalent:

(i) $\left(C L(X), \tau_{w(d)}\right)$ is strongly $\alpha$-favorable for each compatible metric $d$ on $X$,

(ii) $\left(C L(X), \tau_{w(d)}\right)$ is strongly Choquet for each compatible metric $d$ on $X$,

(iii) $X$ is completely metrizable.

Proof. (iii) $\Rightarrow$ (i) holds for any metrizable $X$ (see [26, Theorem 2]), and (i) $\Rightarrow$ (ii) is trivial.

(ii) $\Rightarrow$ (iii) Without loss of generality, assume that $X$ is a locally separable, non-separable metrizable space. Then $X=$ $\bigoplus_{i \in I} X_{i}$ where each $X_{i}$ is separable [14, 4.4.F(c)], so there is a compatible, bounded by $\frac{1}{2}$, totally bounded metric $d_{i}$ on $X_{i}$ for each $i \in I$. If $\left(x_{0}, U_{0}\right) \in \mathcal{E}(X)$ is $\beta$ 's first step in $C h(X)$, then $x_{0} \in X_{i_{0}}$ for some $i_{0} \in I$. Define a compatible metric on $X$ via

$$
d(x, y)= \begin{cases}d_{i}(x, y), & \text { if } x, y \in X_{i}, i \in I, \\ 1, & \text { if } x \in X_{i}, y \in X_{j}, \text { with } i \neq j, i_{0} \notin\{i, j\}, \\ 2, & \text { otherwise. }\end{cases}
$$

Let $\sigma_{w}$ be a winning strategy for $\alpha$ in $\operatorname{Ch}\left(C L(X), \tau_{w(d)}\right)$. For each $S(x, \varepsilon) \in O B\left(X_{i_{0}}\right)$, fix a finite set $F(x, \varepsilon) \subseteq X_{i_{0}} \backslash S(x, \varepsilon)$ such that

$$
X_{i_{0}} \backslash S(x, \varepsilon) \subseteq \bigcup_{z \in F(x, \varepsilon)} B\left(z, \frac{\varepsilon}{2}\right) .
$$

Fix $y \in X \backslash X_{i_{0}}$. Given $k<\omega$, and $G_{k}=\left(\left(x_{0}, U_{0}\right), \ldots,\left(x_{k}, U_{k}\right)\right) \in \mathcal{E}\left(U_{0}\right)^{k+1}$, find $\varepsilon\left(G_{k}\right) \leqslant \frac{1}{2}$ with $S\left(x_{k}, \varepsilon\left(G_{k}\right)\right) \subseteq U_{k}$, and define

$$
\mathbf{G}_{k}=\left[S\left(x_{k}, \frac{\varepsilon\left(G_{k}\right)}{2}\right)\right]^{-} \cap\left\{A \in C L(X): d(y, A)>\frac{3}{2}\right\} \cap \bigcap_{z \in F\left(x_{k}, \varepsilon\left(G_{k}\right)\right)}\left\{A \in C L(X): d(z, A)>\frac{\varepsilon\left(G_{k}\right)}{2}\right\} .
$$


If $\sigma_{w}\left(\left(\left\{x_{0}\right\}, \mathbf{G}_{0}\right), \ldots,\left(\left\{x_{k}\right\}, \mathbf{G}_{k}\right)\right)=W^{-} \cap \bigcap_{j \leqslant m}\left\{A \in C L(X): d\left(y_{j}, A\right)>\varepsilon_{j}\right\}$ for some $y_{j} \in X, \varepsilon_{j}>0$, and $X$-open $W$, then

$$
\bigcup_{z \in F\left(x_{k}, \varepsilon\left(G_{k}\right)\right)} B\left(z, \frac{\varepsilon\left(G_{k}\right)}{2}\right) \subseteq \bigcup_{j \leqslant m} B\left(y_{j}, \varepsilon_{j}\right),
$$

and $x_{k} \in W \subseteq S\left(x_{k}, \frac{\varepsilon\left(G_{k}\right)}{2}\right)$. Then there exists $\delta>0$ such that $S\left(x_{k}, \delta\right) \subseteq W$, and we can put $\sigma\left(G_{k}\right)=S\left(x_{k}, \delta\right)$. Now, if $\alpha$ plays according to $\sigma$ in $\operatorname{Ch}(X)$, then the play

$$
\left(\left\{x_{0}\right\}, \mathbf{G}_{0}\right), \sigma_{w}\left(\left\{x_{0}\right\}, \mathbf{G}_{0}\right), \ldots,\left(\left\{x_{k}\right\}, \mathbf{G}_{k}\right), \sigma_{w}\left(\left(\left\{x_{0}\right\}, \mathbf{G}_{0}\right), \ldots,\left(\left\{x_{k}\right\}, \mathbf{G}_{k}\right)\right), \ldots
$$

generated in $\operatorname{Ch}\left(\operatorname{CL}(X), \tau_{w(d)}\right)$ is compatible with $\sigma_{w}$, so there exists some $A \in \bigcap_{k<\omega} \mathbf{G}_{k}$. It follows that for all $k \geqslant 1$,

$$
A \subseteq X_{i_{0}} \backslash \bigcup_{z \in F\left(x_{k}, \varepsilon\left(G_{k}\right)\right)} B\left(z, \frac{\varepsilon\left(G_{k}\right)}{2}\right) \subseteq S\left(x_{k}, \varepsilon\left(G_{k}\right)\right) \subseteq U_{k} \subseteq \sigma\left(G_{k-1}\right) ;
$$

thus, $\bigcap_{k} \sigma\left(G_{k}\right) \neq \emptyset$, so $X$ is strongly Choquet, and by Choquet's theorem, $X$ is completely metrizable.

It follows from example [6, Example 3.4] that in Theorem B-C we cannot completely remove separability, we give a direct proof here:

Example 4.2. There is the 1 st category metric space $(X, d)$, such that $\left(C L(X), \tau_{w(d)}\right)$ is strongly $\alpha$-favorable.

Proof. Let $X$ be the eventually zero sequences from $\omega_{1}^{\omega}$, where $\omega_{1}$ has the discrete topology. For every $s \in \omega_{1}^{<\omega}$ denote by $|s|$ the cardinality of $s$, and put $[s]=\{f \in X: s \subset f\}$. This topology is metrizable by the Baire metric $d(f, g)=1 / \min \{n+1$ : $f(n) \neq g(n)\}$ for $f, g \in X$. Also, if $f \in[s]$, then $[s]=B\left(f, \frac{1}{|s|+1}\right)$. The set $X_{n}=\{f \in X: \exists i \geqslant n f(i)>0\}$ is open and dense for each $n<\omega$, so $X=\bigcup_{n<\omega} X_{n}^{c}$ is of 1st category. Notice, that

$$
\left(B\left(f, \frac{1}{k}\right)^{c}\right)^{+}=\left\{A \in C L(X): d(f, A)>\frac{1}{k}\right\}
$$

for each $k>0$; thus, $\tau_{b(d)}=\tau_{w(d)}$, so it suffices to prove that $\left(C L(X), \tau_{b(d)}\right)$ is strongly $\alpha$-favorable: let $\mathbf{V} \in \mathcal{B}_{d}$ and $A \in \mathbf{V}$. A winning tactic $t$ for $\alpha$ can be defined by putting $t(A, \mathbf{V})=\mathbf{V}$. To see this, let $\left(A_{0}, \mathbf{V}_{0}\right), \mathbf{V}_{0}, \ldots,\left(A_{k}, \mathbf{V}_{k}\right), \mathbf{V}_{k}, \ldots$ be a play of the strong Choquet game in $\left(C L(X), \tau_{b(d)}\right)$ compatible with $t$. Denote

$$
\mathbf{V}_{k}=\left\langle\left[s_{k, 0}\right], \ldots,\left[s_{k, n_{k}}\right]\right\rangle_{B_{k}}
$$

where $B_{k}=\bigcup_{j \leqslant m_{k}}\left[b_{k, j}\right], s_{k, i}, b_{k, j} \in \omega_{1}^{<\omega}$.

Claim. If $s, b_{n} \in \omega_{1}^{<\omega}$ with $b_{n} \nsubseteq s$ for each $n<\omega$, then $[s] \backslash \bigcup_{n<\omega}\left[b_{n}\right] \neq \emptyset$.

Indeed, if $f \in X$ is defined as

$$
f(m)= \begin{cases}s(m), & \text { if } m<|s|, \\ 1+\sup \left\{\bigcup_{n<\omega} \operatorname{ran} b_{n}\right\}, & \text { if } m=|s|, \\ 0, & \text { if } m>|s|,\end{cases}
$$

then $f \in[s] \backslash \bigcup_{n<\omega}\left[b_{n}\right]$.

To complete the proof, denote $B=\bigcup_{k<\omega} B_{k}$ and, using the Claim, pick $f_{k, i} \in\left[s_{k, i}\right] \backslash B$ for each $k<\omega$ and $i \leqslant n_{k}$. Since $B$ is open in $X$, the $X$-closure $F$ of $\left\{f_{k, i}: k<\omega, i \leqslant n_{k}\right\}$ is disjoint from $B$. It follows, that $F \in \bigcap_{k<\omega} \mathbf{V}_{k}$.

\section{5. (Weak) $\alpha$-favorability of the Wijsman topology}

Theorem 5.1. The following are equivalent:

(i) $\left(C L(X), \tau_{w(d)}\right)$ is (weakly) $\alpha$-favorable,

(ii) $\left(C L(X), \tau_{b(d)}\right)$ is (weakly) $\alpha$-favorable.

Proof. Proposition 3.1 applies, since the spaces are feebly homeomorphic, as can be seen using the proof of [28, Theorem 1.1].

The following is a consequence of [27, Theorem 4.3] (recall that weak $\alpha$-favorability and $\alpha$-favorability coincide in metrizable spaces): 
Proposition 5.2. If $(X, d)$ is (weakly) $\alpha$-favorable, then $\left(C L(X), \tau_{w(d)}\right)$ is $\alpha$-favorable.

A quasi-regular space $X$ (i.e. for each nonempty open $U \subseteq X$ there is a nonempty open $V \subseteq X$ with $\bar{V} \subseteq U$ ) is almost countably subcompact [18], if there is a $\pi$-base $\mathcal{P}$ such that $\bigcap_{n} B_{n} \neq \emptyset$ whenever $\left(B_{n}\right)$ is a sequence of elements of $\mathcal{P}$ with $\overline{B_{n+1}} \subseteq B_{n}$ for all $n<\omega$. So if $B \in \mathcal{P}$ and $t(B) \in \mathcal{P}$ is such that $\overline{t(B)} \subseteq B$, then $t: \mathcal{P} \rightarrow \mathcal{P}$ is a winning tactic for $\alpha$ in $B M(X)$, and hence $X$ is $\alpha$-favorable. Conversely, if $X$ is metrizable and (weakly) $\alpha$-favorable, then it contains a dense completely metrizable subspace [23]; thus, by [1,4.1.3, Theorem 2], $X$ is almost countably subcompact. It follows, that in metrizable spaces almost countable subcompactness (resp. other almost countable Amsterdam properties defined in [1]) is equivalent to (weak) $\alpha$-favorability.

It has been shown in [6, Theorem 2.3], that the separable 1st category metric space $X$ defined in [28, Example 2.5] (see [29] as well) has an almost countably subcompact Wijsman hyperspace. Since this hyperspace is metrizable (because $X$ is separable - cf. [4]), we can get an alternative proof of [6, Theorem 2.3] by showing that $\left(C L(X), \tau_{w(d)}\right)$ is weakly $\alpha$ favorable. The proof is simpler, moreover, it also implies that $\left(C L(X), \tau_{w(d)}\right)$ is almost countably base-compact (this being one of the almost Amsterdam properties equivalent to (weak) $\alpha$-favorability in metrizable spaces), which answers in the positive Question 4.2 of [6]:

Example 5.3. There exists a separable 1 st category metrizable space $X$ with compatible metrics $d$ and $\rho$ such that $\left(C L(X), \tau_{w(d)}\right)$ is $\alpha$-favorable, and $\left(C L(X), \tau_{w(\rho)}\right)$ is $\beta$-favorable.

Proof. Consider $\omega^{\omega}$ with the Baire metric $e(x, y)=1 / \min \{n+1: x(n) \neq y(n)\}$, and its 1 st category subset $\omega^{<\omega}$ of sequences eventually equal to zero. The product $X=\omega^{<\omega} \times \omega^{\omega}$ is a separable, 1 st category space endowed with the metric $d\left(\left(x_{0}, x_{1}\right),\left(y_{0}, y_{1}\right)\right)=\max \left\{e\left(x_{0}, y_{0}\right), e\left(x_{1}, y_{1}\right)\right\}$. Let $p_{1}, p_{2}$ be the projection of $X$ onto $\omega^{<\omega}$ and $\omega^{\omega}$, respectively.

Using Theorem 5.1, we just need to show that $\left(C L(X), \tau_{b(d)}\right)$ is weakly $\alpha$-favorable, since in a metrizable space it is equivalent to $\alpha$-favorability. Inductively define a strategy $\sigma$ for $\alpha$ in $B M\left(C L(X), \mathcal{B}_{d}\right)$ : let $n<\omega$, and assume that

$$
\sigma\left(\mathbf{V}_{0}, \ldots, \mathbf{V}_{i}\right)=\left(B_{i}^{c}\right)^{+} \cap \bigcap_{u \in F_{i}} s\left(u, \frac{1}{m_{i}}\right)^{-}
$$

has been defined for $\left(\mathbf{V}_{0}, \ldots, \mathbf{V}_{i}\right) \in \mathcal{B}_{d}^{i+1}$, where $i \leqslant n, F_{i} \subset X$ is finite, $m_{i} \geqslant i+1, B_{i}$ is a finite union of members of $C B(X)$, and $\left\{S\left(u, \frac{1}{m_{i}}\right): u \in F_{i}\right\}$ is pairwise disjoint and missing $B_{i}$ so that if $i<n$ and $u \in F_{i}$, then there is a $u^{\star} \in F_{i+1}$ such that $p_{1}(u)=p_{1}\left(u^{\star}\right)$ and $d\left(u, u^{\star}\right)<\frac{1}{i+1}$.

The inductive step is analogous to the one made in [29], we sketch the idea for readability: let $\mathbf{V}_{n+1}=\langle\mathcal{V}\rangle_{B_{n+1}} \in \mathcal{B}_{d}$ be such that $\mathbf{V}_{n+1} \subseteq \sigma\left(\mathbf{V}_{0}, \ldots, \mathbf{V}_{n}\right)$, where $\mathcal{V}$ is a finite pairwise disjoint collection of $X$-open balls missing $B_{n+1}$, which, without loss of generality, is the union of the finite pairwise disjoint collection $\left\{S\left(b_{j}, \frac{1}{n_{j}}\right): j \in J\right\}$ of clopen $X$-balls. Let $A \in \mathbf{V}_{n+1}$ be finite, and $u \in F_{n}$. If $u \notin B_{n+1}$, choose $u^{\star}=u$. If, on the other side, $u \in S\left(b_{j_{0}}, \frac{1}{n_{j_{0}}}\right)$ for some $j_{0} \in J$, then $n_{j_{0}}>m_{n}$. Choose $k \in \omega \backslash\left\{p_{2}\left(b_{j}\right)\left(m_{n}\right): j \in J\right\}$, define $u_{2} \in \omega^{\omega}$ via

$$
u_{2}(s)= \begin{cases}p_{2}(u)(s), & \text { if } s \neq m_{n}, \\ k, & \text { if } s=m_{n},\end{cases}
$$

and put $u^{\star}=\left(p_{1}(u), u_{2}\right)$. To complete the induction, let $F_{n+1}=A \cup\left\{u^{\star}: u \in F_{n}\right\}$, and find $m_{n+1} \geqslant n+2$ so that

$$
\sigma\left(\mathbf{V}_{0}, \ldots, \mathbf{V}_{n+1}\right)=\left(B_{n+1}^{c}\right)^{+} \cap \bigcap_{u \in F_{n+1}} s\left(u, \frac{1}{m_{n+1}}\right)^{-} \subseteq \mathbf{V}_{n+1} .
$$

To show that $\sigma$ is a winning strategy for $\alpha$, let $\mathbf{V}_{0}, \mathbf{U}_{0}, \ldots, \mathbf{V}_{n}, \mathbf{U}_{n}, \ldots$ be a play of the Banach-Mazur game on $C L(X)$ compatible with $\sigma$, i.e. $\mathbf{U}_{n}=\sigma\left(\mathbf{V}_{0}, \ldots, \mathbf{V}_{n}\right)$ for each $n$. Whenever $u \in F_{i}$ for some $i \geqslant 1$, the sequence $u, u^{\star}, u^{\star \star}, \ldots$ is a Cauchy sequence in $\left\{p_{1}(u)\right\} \times \omega^{\omega}$; hence, it converges to some $u^{\infty} \in S\left(u, \frac{1}{m_{i}}\right)$. Because the $B_{i}$ 's are disjoint from the

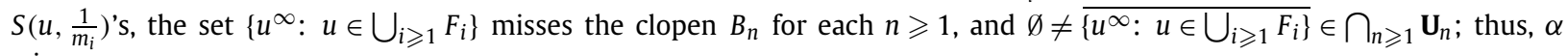
wins.

If $\left(C L(X), \tau_{w(\rho)}\right)$ were a Baire space for every compatible metric $\rho$ on (the separable) $X$, then $X$ would be a Baire space by [28, Theorem 2.3], so there must be a compatible metric $\rho$ such that $\left(C L(X), \tau_{w(\rho)}\right)$ is not a Baire space, and therefore $\left(C L(X), \tau_{w(\rho)}\right)$ is $\beta$-favorable.

It follows from the above example that Proposition 5.2 cannot be reversed. There is however an other way of looking at $\alpha$-favorability of the Wijsman hyperspace. Since (weak) $\alpha$-favorability is a topological property, we can rephrase Proposition 5.2 as follows:

Proposition 5.4. If $X$ is (weakly) $\alpha$-favorable, then $\left(C L(X), \tau_{w(d)}\right)$ is $\alpha$-favorable for each compatible metric $d$ on $X$. 
It would be reasonable to think that the converse of the previous proposition is true for at least a separable $X$, since the analogous result for the strong Choquet game holds (Theorem 4.1), and non- $\beta$-favorability of $\left(C L(X), \tau_{w(d)}\right)$ for every compatible metric $d$ on $X$ is necessary for non- $\beta$-favorability of $X$ [28, Theorem 2.3]. Our last result shows however, that this is not true:

Theorem 5.5. There exists a separable non- $\alpha$-favorable metrizable space $X$ such that $\left(C L(X), \tau_{w(d)}\right)$ is $\alpha$-favorable for each compatible metric $d$ on $X$.

Proof. Let $X \subset \mathbb{R}$ be a Bernstein set, i.e. both $X$ and $\mathbb{R} \backslash X$ intersect each dense-in-itself $G_{\delta}$-subset of $\mathbb{R}$. It is well known, that $X$ is not $\alpha$-favorable [17], since otherwise $X$ would contain a dense completely metrizable subspace, and hence, a dense-initself $G_{\delta}$ (for an alternative proof see [21]).

In what follows, $\bar{A}$ will stand for the closure of $A \subseteq \mathbb{R}$ in $\mathbb{R}$. Let $d$ be a compatible metric on $X$. In view of Theorem 5.1, it suffices to define a winning tactic $t$ for $\alpha$ in $B M\left(C L(X), \tau_{b(d)}\right)$ : first, let $\mathcal{B}^{\prime}$ be the collection of bounded open intervals in $\mathbb{R}$, and $\mathcal{B}=\left\{V^{\prime} \cap X: V^{\prime} \in \mathcal{B}^{\prime}\right\}$. Then for any $V=V^{\prime} \cap X \in \mathcal{B}, V^{\prime}=\operatorname{int}(\bar{V})$ and for $U, V \in \mathcal{B}, U \subseteq V$ iff $U^{\prime} \subseteq V^{\prime}$. The collection

$$
\mathcal{P}_{d}=\left\{\langle\mathcal{V}\rangle_{B} \in \mathcal{B}_{d}: \delta=\operatorname{dist}_{d}(\bigcup \mathcal{V}, B)>0\right\}
$$

is a $\pi$-base of $\left(C L(X), \tau_{b(d)}\right)$. Let $\langle\mathcal{V}\rangle_{B} \in \mathcal{P}_{d}$, where $\mathcal{V}=\left\{V_{0}, \ldots, V_{n}\right\}, B=\bigcup_{j \leqslant p} B\left(z_{j}, \varepsilon_{j}\right)$, and denote $D=\bigcup_{j \leqslant p} B\left(z_{j}, \varepsilon_{j}+\frac{\delta}{2}\right)$. Note that

$$
a \notin D \text { implies } d(a, B) \geqslant \frac{\delta}{2} \text {. }
$$

For each $i \leqslant n$ define disjoint $U_{i, 0}^{\prime}, U_{i, 1}^{\prime} \in \mathcal{B}^{\prime}$ such that for $r=0,1$,

$$
\overline{U_{i, r}^{\prime}} \subseteq V_{i}^{\prime} \text { and } \operatorname{length}\left(U_{i, r}^{\prime}\right) \leqslant \frac{1}{2} \operatorname{length}\left(V_{i}^{\prime}\right)
$$

Put $t\left(\langle\mathcal{V}\rangle_{B}\right)=\left\langle U_{0,0}, U_{0,1}, \ldots, U_{n, 0}, U_{n, 1}\right\rangle_{D}$.

Let $\mathbf{V}_{0}, t\left(\mathbf{V}_{0}\right), \ldots, \mathbf{V}_{k}, t\left(\mathbf{V}_{k}\right), \ldots$ be a run of $B M\left(C L(X), \tau_{b(d)}\right)$ compatible with $t$. Let $\mathbf{V}_{0}=\left\langle\mathcal{V}_{0}\right\rangle_{B_{0}} \in \mathcal{P}_{d}$, where $\mathcal{V}_{0}=$ $\left\{V_{0}^{0}, \ldots, V_{n_{0}}^{0}\right\}$ for some $n_{0}<\omega$. Then $t\left(\mathbf{V}_{0}\right)=\left\langle\mathcal{U}_{0}\right\rangle_{D_{0}}$ where $\mathcal{U}_{0}=\left\{U_{0,(0)}^{0}, U_{0,(1)}^{0}, \ldots, U_{n_{0},(0)}^{0}, U_{n_{0},(1)}^{0}\right\}$, and by Theorem 2.1, we can label $\mathbf{V}_{1}=\left\langle\mathcal{V}_{1}\right\rangle_{B_{1}} \in \mathcal{P}_{d}$ so that

$$
\mathcal{V}_{1}=\left\{V_{0,(0)}^{0}, V_{0,(1)}^{0}, \ldots, V_{n_{0},(0)}^{0}, V_{n_{0},(1)}^{0}, V_{0}^{1}, \ldots, V_{n_{1}}^{1}\right\} \text {, }
$$

where $n_{1}<\omega, V_{i, s}^{0} \subseteq U_{i, s}^{0}$ for all $i \leqslant n_{0}, s \in\{0,1\}$, and $B_{1} \supseteq D_{0}$. Then $t\left(\mathbf{V}_{1}\right)=\left\langle\mathcal{U}_{1}\right\rangle_{D_{1}}$, where

$$
\mathcal{U}_{1}=\left\{U_{0,(00)}^{0}, U_{0,(01)}^{0}, U_{0,(10)}^{0}, U_{0,(11)}^{0}, \ldots, U_{n_{0},(10)}^{0}, U_{n_{0},(11)}^{0}, U_{0,(0)}^{1}, U_{0,(1)}^{1}, \ldots, U_{n_{1},(0)}^{1}, U_{n_{1},(1)}^{1}\right\},
$$

and we can label $\mathbf{V}_{2}=\left\langle\mathcal{V}_{2}\right\rangle_{B_{2}} \in \mathcal{P}_{d}$ so that

$$
\mathcal{V}_{2}=\left\{V_{0,(00)}^{0}, V_{0,(01)}^{0}, \ldots, V_{n_{0},(10)}^{0}, V_{n_{0},(11)}^{0}, V_{0,(0)}^{1}, V_{0,(1)}^{1}, \ldots, V_{n_{1},(0)}^{1}, V_{n_{1},(1)}^{1}, V_{0}^{2}, \ldots, V_{n_{2}}^{2}\right\},
$$

where $n_{2}<\omega, V_{i, s}^{j} \subseteq U_{i, s}^{j}$ for all $j<2, i \leqslant n_{j}, s \in\{0,1\}^{2-j}$, and $B_{2} \supseteq D_{1}$. Continuing this labeling, for each $k \geqslant 1$ we can write $\mathbf{V}_{k}=\left\langle\mathcal{V}_{k}\right\rangle_{B_{k}} \in \overline{\mathcal{P}}_{d}$, so that

$$
\mathcal{V}_{k}=\bigcup_{j \leqslant k} \bigcup_{i \leqslant n_{j}}\left\{V_{i, s}^{j}: s \in\{0,1\}^{k-j}\right\} \quad\left(\text { when } j=k \text {, write } V_{i}^{k} \text { instead of } V_{i, \emptyset}^{k}\right),
$$

and if $\left\langle\mathcal{U}_{k-1}\right\rangle_{D_{k-1}}=t\left(\mathbf{V}_{k-1}\right)$, where

$$
\mathcal{U}_{k-1}=\bigcup_{j<k} \bigcup_{i \leqslant n_{j}}\left\{U_{i, s}^{j}: s \in\{0,1\}^{k-j}\right\},
$$

then $V_{i, s}^{j} \subseteq U_{i, s}^{j}$ for all $j<k, i \leqslant n_{j}, s \in\{0,1\}^{k-j}$, and $B_{k} \supseteq D_{k-1}$. It follows by (2) that, for a given $k<\omega, i \leqslant n_{k}$, and $s \in\{0,1\}^{\omega}, \bigcap_{m<\omega} \overline{\left(V_{i, S_{m}}^{k}\right)^{\prime}}$ is a singleton, and

$$
A_{k}=\bigcap_{m<\omega} \overline{\bigcup\left\{\left(V_{i, s}^{k}\right)^{\prime}: i \leqslant n_{k}, s \in\{0,1\}^{m}\right\}}
$$

is a closed dense-in-itself subset of $\mathbb{R}$ for each $k<\omega$. Then $A=\bigcup_{k<\omega} A_{k}$ is also dense-in-itself, and so is $\bar{A}$. Moreover, if an $\mathbb{R}$-open $W$ hits $\bar{A}$, or $A_{k}$, respectively, then $\bar{A} \cap W, A_{k} \cap W$, respectively, is a dense-in-itself $G_{\delta}$ subset of $\mathbb{R}$; thus, by the definition of the Bernstein set, 


$$
\begin{aligned}
& \bar{A} \cap W \cap X \neq \emptyset, \quad \text { and } \\
& A_{k} \cap W \cap X \neq \emptyset, \quad \text { respectively. }
\end{aligned}
$$

In particular, (3) implies that $F=\bar{A} \cap X \neq \emptyset$, i.e. $F \in C L(X)$. Furthermore, if $F \cap W \neq \emptyset$, then $A \cap W \neq \emptyset$, so $A_{k} \cap W \neq \emptyset$ for some $k$, and by (4), $A_{k} \cap W \cap X \neq \emptyset$; thus, $A \cap X$ is dense in $F$.

Fix $k<\omega$, and $V \in \mathcal{V}_{k}$. Then $V=V_{i, s}^{j}$ for some $j \leqslant k, i \leqslant n_{j}$, and $s \in\{0,1\}^{k-j}$, so if $q \in\{0,1\}^{\omega}$ is such that $\left.q\right|_{k-j+1}=s$, then for all $m \geqslant k-j+1$,

$$
\overline{\left(V_{i, q\rceil_{m}}^{j}\right)^{\prime}} \subseteq \overline{\left(U_{i,\left.q\right|_{m}}^{j}\right)^{\prime}} \subseteq\left(V_{i, s}^{j}\right)^{\prime}=V^{\prime},
$$

so $\emptyset \neq A_{j} \cap V^{\prime} \subseteq \bar{A} \cap V^{\prime}$, and by (3), $F \cap V=\bar{A} \cap V^{\prime} \cap X \neq \emptyset$; thus, $F \in V^{-}$.

We will now show that $F \cap B_{k}=\emptyset$ : let $x \in F$. Since $A \cap X$ is dense in $F$, we can find a sequence $a_{m} \in A \cap X$ that $d$ converges to $x$. For each $m<\omega$ denote $l_{m}=\min \left\{l \geqslant k+1: a_{m} \in \bigcup \mathcal{V}_{l}\right\}$. Then $a_{m} \in \bigcup \mathcal{V}_{l_{m}}$, so $a_{m} \notin B_{l_{m}} \supseteq D_{k}$, and consequently, by (1), $d\left(a_{m}, B_{k}\right) \geqslant \frac{\delta_{k}}{2}$ for each $m$, where $\delta_{k}=\operatorname{dist}_{d}\left(\cup \mathcal{V}_{k}, B_{k}\right)$. Then $d\left(x, B_{k}\right) \geqslant \frac{\delta_{k}}{2}>0$, so $x \notin B_{k}$. It follows that $F \in \bigcap_{k} \mathbf{V}_{k}$.

Remark 5.6. The previous example suggests the following open problem:

Does there exist a metrizable non-Baire space $X$ such that $\left(C L(X), \tau_{w(d)}\right)$ is a Baire space for each compatible metric $d$ on $X$ ?

Note that due to [28, Theorem 2.3], if such a space exists, the set of points of local separability is not dense in $X$.

\section{References}

[1] D.J. Lutzer, Completeness properties designed for recognizing Baire spaces, Dissertationes Math. 116 (1974) 1-43.

[2] A. Barbati, The hyperspace of an analytic metrizable space is analytic, Rend. Istit. Mat. Univ. Trieste 25 (1993) 15-21.

[3] G. Beer, A Polish topology for the closed subsets of a Polish space, Proc. Amer. Math. Soc. 113 (1991) 1123-1133.

[4] G. Beer, Topologies on Closed and Closed Convex Sets, Kluwer, Dordrecht, 1993.

[5] G. Choquet, Lectures on Analysis I, Benjamin, New York, 1969.

[6] J. Cao, H.J.K. Junnila, Amsterdam properties of Wijsman hyperspaces, Proc. Amer. Math. Soc. 138 (2010) 769-776.

[7] J. Cao, A.H. Tomita, The Wijsman hyperspace of a metric hereditarily Baire space is Baire, Topology Appl. 157 (2010) 145-151.

[8] J. Chaber, R. Pol, Note on the Wijsman hyperspaces of completely metrizable spaces, Boll. Unione Mat. Ital. Sez. B Artic. Ric. Mat. (8) 5 (2002) $827-832$.

[9] C. Costantini, Every Wijsman topology relative to a Polish space is Polish, Proc. Amer. Math. Soc. 123 (1995) 2569-2574.

[10] C. Costantini, On the hyperspace of a non-separable metric space, Proc. Amer. Math. Soc. 126 (1998) 3393-3396.

[11] C. Costantini, S. Levi, J. Pelant, Infima of hyperspace topologies, Mathematica 42 (1995) 67-86.

[12] G. Debs, Stratégies gagnantes dans certains jeux topologiques, Fund. Math. 126 (1985) 93-105.

[13] E.G. Effros, Convergence of closed subsets in a topological space, Proc. Amer. Math. Soc. 16 (1965) 929-931.

[14] R. Engelking, General Topology, Helderman, Berlin, 1989.

[15] F. Galvin, R. Telgársky, Stationary strategies in topological games, Topology Appl. 22 (1986) 51-69.

[16] L'. Holá, R. Lucchetti, Equivalence among hypertopologies, Set-Valued Anal. 3 (1995) 339-350.

[17] R.C. Haworth, R.A. McCoy, Baire spaces, Dissertationes Math. 141 (1977) 1-77.

[18] Y. Ikeda, Čech-completeness and countable subcompactness, Topology Proc. 14 (1989) 75-87.

[19] A.S. Kechris, Classical Descriptive Set Theory, Springer, New York, 1994.

[20] E. Michael, Topologies on spaces of subsets, Trans. Amer. Math. Soc. 71 (1951) 152-182.

[21] L. Piątkiewicz, L. Zsilinszky, On (strong) $\alpha$-favorability of the Vietoris hyperspace, preprint.

[22] R. Telgársky, Topological games: On the 50th anniversary of the Banach-Mazur game, Rocky Mountain J. Math. 17 (1987) $227-276$.

[23] H.E. White, Topological spaces that are a-favorable for a player with perfect information, Proc. Amer. Math. Soc. 50 (1975) 477-482.

[24] R. Wijsman, Convergence of sequences of convex sets, cones and functions II, Trans. Amer. Math. Soc. 123 (1966) 32-45.

[25] L. Zsilinszky, Baire spaces and hyperspace topologies, Proc. Amer. Math. Soc. 124 (1996) 2575-2584.

[26] L. Zsilinszky, Polishness of the Wijsman topology revisited, Proc. Amer. Math. Soc. 126 (1998) 3763-3765.

[27] L. Zsilinszky, Topological games and hyperspace topologies, Set-Valued Anal. 6 (1998) 187-207.

[28] L. Zsilinszky, On Baireness of the Wijsman Hyperspace, Boll. Unione Mat. Ital. Sez. B Artic. Ric. Mat. (8) 10 (2007) 1071-1079.

[29] L. Zsilinszky, Corrigendum to "On Baireness of the Wijsman Hyperspace”, Boll. Unione Mat. Ital. (9) II (2009) 575-577. 